\title{
Frailty and Loss of Rectus Femoris Muscle Thickness in Hospitalized Elderly Patients with Community-Acquired Pneumonia - A Case-Control Study
}

\author{
Hirokazu Touge ${ }^{1}$, Katsuyuki Tomita ${ }^{1,}{ }^{*}$, Shizuka Nishii ${ }^{1}$, Tsuyoshi Kitaura ${ }^{1,2}$, Yasuto Ueda ${ }^{1,2}$, \\ Keiji Matsunami $^{3}$, Hiromitsu Sakai ${ }^{1}$, Akira Yamasaki ${ }^{2}$, Yuji Kawasaki ${ }^{2}$, Eiji Shimizu ${ }^{2}$ \\ ${ }^{1}$ Department of Respiratory Medicine, Yonago Medical Centre, Tottori, Japan \\ ${ }^{2}$ Division of Medical Oncology and Molecular Respirology, Tottori University Faculty of Medicine, Tottori, Japan \\ ${ }^{3}$ Department of Palliative Care Medicine, Yonago Medical Centre, Tottori, Japan
}

Email address:

tomita-katsuyuki@yonagomc.jp (K. Tomita)

${ }^{*}$ Corresponding author

\section{To cite this article:}

Hirokazu Touge, Katsuyuki Tomita, Shizuka Nishii, Tsuyoshi Kitaura, Yasuto Ueda, Keiji Matsunami, Hiromitsu Sakai, Akira Yamasaki, Yuji Kawasaki, Eiji Shimizu. Frailty and Loss of Rectus Femoris Muscle Thickness in Hospitalized Elderly Patients with CommunityAcquired Pneumonia - A Case-Control Study. Clinical Medicine Research. Vol. 6, No. 3, 2017, pp. 86-91. doi: 10.11648/j.cmr.20170603.15

Received: February 27, 2017; Accepted: March 20, 2017; Published: April 11, 2017

\begin{abstract}
Frailty is at the core of geriatric syndromes that are characterized by multiple morbidities and mobility limitations. The purpose of this case-control study was to assess the frailty scale and muscle mass on the acute phase in hospitalized elderly patients with community-acquired pneumonia (CAP). Thirty-two elderly patients, aged 75 years or older, who were hospitalized with CAP, and 32 control patients hospitalized without CAP were recruited. Frailty scale and muscle mass were assessed using the Reported Edmonton Frail Scale (REFS) and rectus femoris (RF) thickness by ultrasound, respectively. There was a significant increase in the frequency of frailty among CAP patients (50\%), compared with control patients $(22 \%)$. Frailty scale correlated inversely with RF thickness and activity status. Based upon the piece-wise linear regression analysis between REFS and RF thickness decreased rapidly with the pre-frailty status among CAP patients. Elderly patients with CAP exhibit a significant impairment of activity status with frailty due to decreased thickness of RF prior to admission.
\end{abstract}

Keywords: Elder, Frailty, Pneumonia, Rectus Femoris

\section{Introduction}

Community-acquired pneumonia (CAP) is a frequent cause of hospitalization and death among the elderly [1]. CAP rates in the elderly are rising as a consequence of the overall increase of the elderly population [2]. The incidence of hospitalized CAP is much higher among elderly patients [3].

Frailty is at the core of geriatric syndromes, which are characterized by disability of morbidity and functional status $[4,5]$. Elderly patients with poor function status, such as frailty, are characterized by a higher risk of developing chronic lower respiratory tract disease [6], and CAP [7, 8]. Furthermore, frailty was associated with recurrent admission for pneumonia [9] and poorer outcomes [10] among elderly patients with CAP.

Major factors leading to frailty include sarcopenia, which is the loss of skeletal muscle mass that can accompany aging, and a decline in executive function. Sarcopenia begins in the fourth decade of life and accelerates after approximately 75 years of age [11]. According to the criteria for sarcopenia established by the European Working Group on Sarcopenia in Older People (EWGSOP), affected subjects exhibit three components, low muscle mass, reduced muscle strength and low physical performance [12]. While the typical "gold standard" for assessing muscle mass has been the use of dual energy $\mathrm{x}$-ray absorptiometry, magnetic resonance imaging (MRI) and computerized tomography (CT) have also been used as supplementary tools; however, these processes are very expensive and generally require the subjects to exhibit a mobility-intact state. 
Gait speed and walking abilities, which are among the components of sarcopenia, may be used as clinical indicators of frailty in the elderly $[13,14]$. As it has been reported that decreasing thigh muscle is one of predictors of gait speed decline [15], assessment of rectus femoris (RF) thickness is considered to be useful for detecting physical frailty, substitute for gait speed in the mobility-intact state. We hypothesized that the assessment of muscle mass would be a useful tool to detect the pre-frailty status in the frailty timewindow, which is the time between good performance and disability, in elderly patients hospitalized with CAP. The aim of this case-control study was to investigate the potential association between frailty scale and muscle mass. In this study, patients were enrolled following emergency hospital admission. As the validity and reliability of measurement of the RF muscle diameter using B-mode diagnostic ultrasound (US) in the assessment of muscle mass has been reported [16], we assessed muscle mass by this means in the immobile state.

\section{Method}

\subsection{Study Protocol}

In this case-control study, 32 patients with communityacquired pneumonia (CAP) and 32 sex- and age-matched control patients without CAP were enrolled from August 2012 to June 2013. All patients aged 75 years or older who were hospitalized in respiratory medicine and internal medicine wards at Yonago Medical Centre were screened within 24 hours of admission to determine the frailty scale, muscle mass, activity status of life, and nutritional status. Sex- and age-matched control subjects without CAP were randomly selected in the wards, based on the same inclusion and exclusion criteria. All patients with and without CAP were not disabled (i.e. required no personal assistance) with respect to active walking prior to admission.

As congestive heart failure [17], depression [17], and anemia [18] have been reported as risk factors for frailty, these patients were excluded from this study. Exclusion criteria also included cancer, inability to be interviewed, a past medical history of CAP, or unwillingness to provide informed consent. The study protocol was approved by the ethics committee of Yonago Medical Centre. Signed informed consent was provided by each patient, their next of kin or other surrogates for individuals with cognitive impairment after being informed orally by telephone.

The decision for hospital admission due to CAP was based on the score of the CURB-65 (Confusion, Urea nitrogen, Respiratory rate, Blood pressure, at the age of 65 years and older), and a score of 2 or more according to the British Thoracic Society guideline [19].

\subsection{Assessment on Admission}

\subsubsection{Frailty Scale}

Frailty was defined according to the Reported Edmonton Frail Scale (REFS) [20]. The REFS is a scale that assesses frailty in acute inpatients among the elderly, and evaluates nine domains: cognition, general health status, functional independence, social support, medication use, nutrition, mood, continence and functional performance, through the examination of 11 items. The maximum score is 18 , representing the highest level of frailty. For this analysis, the scores are: 0-7, "not frail"; 8-18, "frail".

\subsubsection{Muscle Mass}

Considering the need for emergency hospital admission and the immobile state, assessment of muscle mass was determined by measuring the thickness of the rectus femoris (RF) using ultrasonography. All measurements were performed by a single experienced investigator (K. T.). RF diameter was measured by B-mode ultrasonography using a wall tracking ultrasound system (Fukuda Denshi, model 4500) and a $5 \mathrm{MHz}$ transducer (FUT-L104). The left thigh was examined as our patients had no history of bone fracture at the left extremity. The transducer was placed $15 \mathrm{~cm}$ above the superior border of the patella following the midline of the anterior surface of the thigh with liberal use of contact gel and minimal pressure to avoid compression of the muscle [21]. Five consecutive images were taken and the average thickness was used as the RF thickness. The vertical thickness of the RF muscle was measured on the inner edge of the muscle (Figure 1). Loss of muscle mass $\leq 10 \mathrm{~mm}$ of the RF thickness was considered to be thin muscle, based upon previous reports that demonstrated the $10 \mathrm{~mm}$ cutoff as $2 \mathrm{SD}$ below the mean of thickness of the RF in the same age bracket [22].
(A)

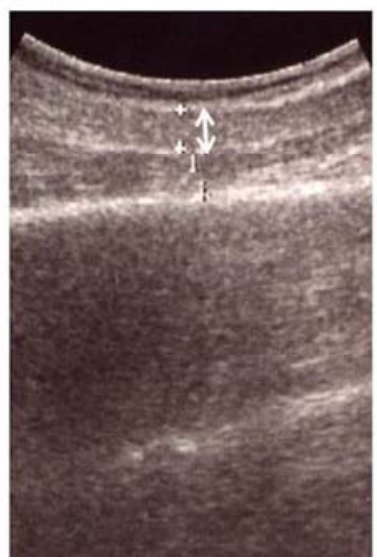

(B)

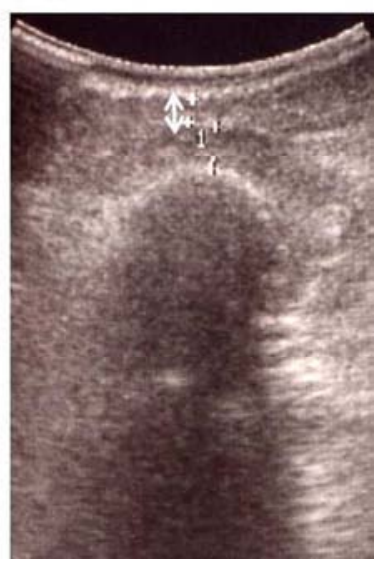

Figure 1. Ultrasonographic images showing the muscle thickness of the rectus femoris $(R F)$ muscle indicating the axial $(A)$ and vertical thickness (B).

\subsubsection{Activity and Nutritional Status}

We determined the activity status using the Eastern Cooperative Oncology Group (ECOG) performance status [23], which was assigned as follows: grade 0; fully active, grade 1; restricted in physically strenuous activity but ambulatory and able to carry out work of a light or sedentary nature, grade 2; ambulatory and capable of all self-care, but unable to carry out any work activities, up to and more than 
$50 \%$ of their waking hours, grade 3 ; capable of only limited self-care, confined to a bed or chair for more than $50 \%$ of their waking hours, grade 4; completely disabled.

We assessed the nutritional status using the quick and easily administered mini nutritional assessment test-short form (MNA-SF) [24]. The maximum MNA-SF score is 14 points, with a score $\leq 7$ points indicating malnutrition, 8-11 points indicating a risk of malnutrition and $12-14$ points indicating that the person has a normal nutritional status.

\subsubsection{Clinical Data}

Clinical data obtained from the patients' charts included biochemical measurements relevant to nutritional status, i.e., albumin, total lymphocyte count (TLC), hemoglobin, WBC and total cholesterol. The tests were performed at the central chemistry lab of Yonago Medical Centre using standard methods.

\subsection{Statistical Analysis}

\section{(A)}

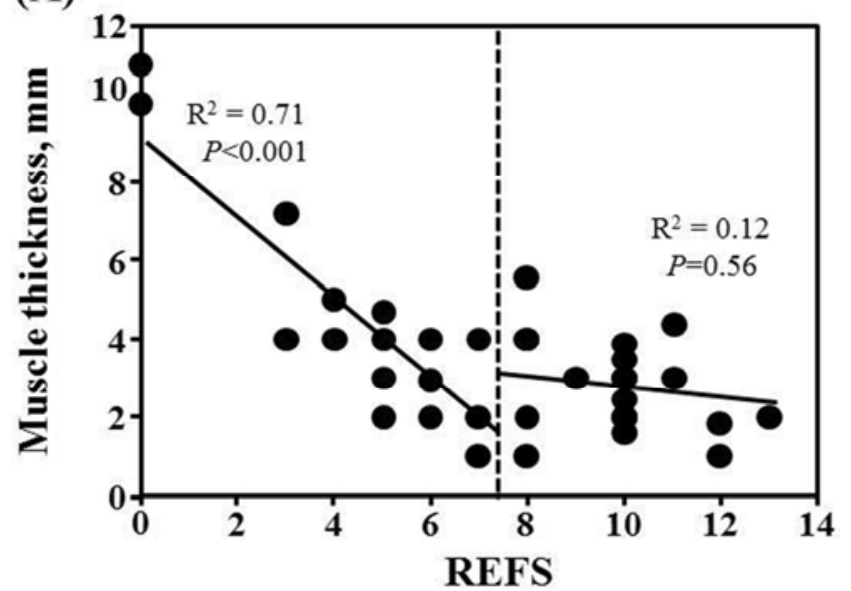

(B)

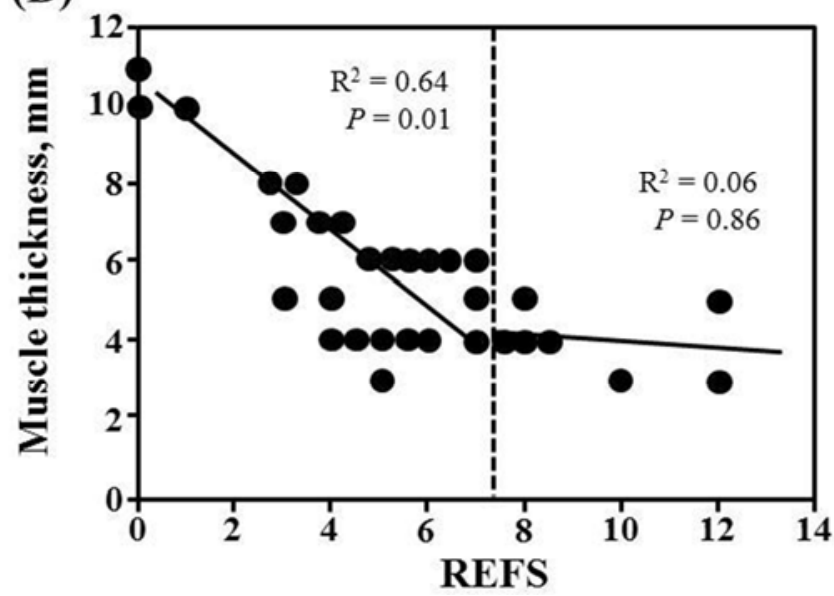

Figure 2. Piece-wise linear regression of the Reported Edmonton Frail Scale (REFS) and RF thickness and that of the REFS in elderly patients with (A) and without $C A P(B)$.

Data were abstracted from the patients' hospital files and computer records. Normality for continuous variables was determined using the Kolmogorov-Smirnov test. As the variables were not normally distributed, differences of continuous variables between the groups were analyzed using the Mann-Whitney U test, whereas categorical variables were analyzed using the $\chi 2$ test (or Fisher's exact test for small proportions). As frailty is present in the elderly general populations in up to $20 \%$ of 75 year old or more [18], we based our sample size calculations on the assumption that frailty would be present in $20 \%$ more of the elderly patients with CAP than the control patients. Under this assumption, a sample of 30 pairs of elderly patients with CAP and controls would be sufficient to detect a difference of this magnitude in the frequency of both groups. To investigate whether there were any associations between frailty scales, muscle mass, and undernutrition, Spearman's non-parametric rank correlation coefficient was calculated. The scatterplots of the REFS and RF thickness show that these correlations were not best fit by a single straight line (Figure 2). The trend of the points was "steeper" for lower values of REFS and "flatter" for higher values of REFS. Thus, the model selected to describe the linear relationship between the REFS and RF thickness was a "segmented" line [25]. Statistical analyses were performed using SPSS software version 19.0 (SPSS Inc., Chicago, Ill., USA). $P$-values less than 0.05 were considered to indicate statistical significance.

\section{Results}

Table 1. Patient characteristics stratified by frailty status.

\begin{tabular}{|c|c|c|c|}
\hline & $\begin{array}{l}\text { Patients with } \\
\text { elderly CAP }\end{array}$ & Controls & $P$-value* \\
\hline $\mathrm{N}$ & 32 & 32 & \\
\hline Age, years ${ }^{\dagger}$ & $83.3 \pm 5.1$ & $82.5 \pm 4.4$ & 0.55 \\
\hline Women, $\%$ & 47 & 56 & 0.45 \\
\hline Activity status of life ${ }^{\dagger}$ & $3.4 \pm 1.1$ & $2.7 \pm 0.8$ & 0.003 \\
\hline $\mathrm{BMI}, \mathrm{kg} / \mathrm{m}^{2 \dagger}$ & $20.2 \pm 3.8$ & $20.5 \pm 3.6$ & 0.78 \\
\hline Calf thickness, $\mathrm{cm}^{\dagger}$ & $26.9 \pm 3.7$ & $26.8 \pm 3.9$ & 0.92 \\
\hline MNA-SF score $^{\dagger}$ & $8.8 \pm 3.4$ & $9.3 \pm 2.8$ & 0.70 \\
\hline RF thickness, $\mathrm{mm}^{\dagger}$ & $3.8 \pm 2.4$ & $5.5 \pm 2.1$ & 0.0004 \\
\hline Albumin, $\mathrm{g} / \mathrm{dL}^{\dagger}$ & $3.1 \pm 0.6$ & $3.1 \pm 0.7$ & 0.96 \\
\hline REFS & $7.2 \pm 3.3$ & $5.5 \pm 2.9$ & 0.01 \\
\hline$\%$ Frail & 50 & 22 & 0.02 \\
\hline Residence $^{\ddagger}$ & & & 0.65 \\
\hline Resides with family & 16 & 18 & \\
\hline Spouse & 7 & 8 & \\
\hline Resides alone & 5 & 5 & \\
\hline Nursing home & 4 & 1 & \\
\hline Co-morbid disease & & & 0.43 \\
\hline None & 8 & 12 & \\
\hline Dementia & 5 & 5 & \\
\hline Osteoarthropathia & 4 & 2 & \\
\hline Cardiovascular disease & 4 & 3 & \\
\hline Diabetes mellitus & 3 & 4 & \\
\hline Cerebral vascular disease & 4 & 6 & \\
\hline Post-fracture & 2 & 1 & \\
\hline COPD & 2 & 1 & \\
\hline Other & 2 & 1 & \\
\hline
\end{tabular}

All variables are reported as the median (quartile of percentile). ${ }^{*} P$-value for differences between CAP and non-CAP patients. $\dagger$ indicates Mann-Whitney rank-sum analysis. $\$$ indicates chi-squared test. Abbreviations: BMI, body mass index; MNA-SF, mini nutritional assessment test-short form; REFS, Reported Edmonton Frail Scale; RF, rectus femoris. 
The baseline characteristics of the patients in both groups are shown in Table 1 . Of 32 patients with gender- and agematched control patients, 18 patients had urinary tract infections, two had transient ischemic attack (TIA), 11 were tested for solitary pulmonary nodules, and one for iliopsoas abscess. Table 1 shows significantly lower activity status of life, lower RF thickness, and higher score of frailty among the elderly patients with CAP compared with controls. There were no significant differences in nutritional status according to the MNA-SF criteria $(P=0.70)$, BMI, or biochemical measurements including albumin, TLC, hemoglobin and total cholesterol between the two groups.

For these analyses, 'non-frail' was defined as REFS 0-7 and 'frail' was defined as REFS 8-18.

Among elderly patients with CAP, $50 \%$ were identified as being frail according to REFS. On the other hand, seven of 32 patients $(22 \%)$ without CAP were frail. The frail patients with CAP had a significantly lower RF thickness $(2.6 \pm 1.2$ $\mathrm{mm})$ compared with the non-frail patients with CAP (4.2 \pm $2.1 \mathrm{~mm})(P=0.03)$.

Of 32 elderly patients with CAP, 25 patients exhibited scores of 2 and seven exhibited scores of 3 for the CURB- 65 . In determining Spearman's correlation coefficient in patients with CAP, RF thickness was associated inversely with both the REFS (rho $=-0.42, P=0.02$ ) and the activity score of life (rho $=-0.42, P=0.02$ ). The REFS was associated positively with frailty MNA-SF (rho $=-0.43, P=0.02$ ) and the activity status of life (rho $=-0.38, P=0.002$ ). There was no association between CURB-65 and the other factors including REFS, activity status of life, and RF thickness.

As the association between the REFS and RF thickness exhibited a piece-wise line (Figure 2), we analyzed this association using segmented regression analysis. In the scatterplots, we found an REFS threshold of 8 points by linear piecewise regression modeling of RF thickness and the trend of the points was "steeper" for lower values of REFS and "flatter" for higher values of REFS in elderly patients with CAP.

\section{Conclusions}

Frailty is a major determinant of adverse health outcomes, such as functional limitations and disability in dwelling populations, as well as in those with diseases, such as CAP. In this study, half of the elderly patients hospitalized with CAP exhibited frailty upon admission. In the pre-status of 'frail', muscle thickness and activity status of life were associated inversely with the Reported Edmonton Frail Scale (REFS).

Elderly patients fail to thrive due to impaired physical function, malnutrition, depression, and cognitive impairment. Our result for the prevalence of frailty in control patients was consistent with the report by Castell and colleagues [26] demonstrated that it was $19.1 \%$ in persons age 75 and older, based on population-based cohorts. Elderly patients with impairment of physical performance are characterized by a higher risk of developing CAP [7, 8]. Our results, which demonstrated that half of the elderly patients hospitalized with CAP exhibited frailty, imply that frailty might be a key to the development of pneumonia in the elderly with low performance status.

Frailty can manifest as several phenotypes [4], one of which, physical frailty, is typified by low muscle mass and low physical performance, which could be assessed using gait speed and muscle strength. Gait speed is easily measured, clinically interpretable, and is potentially modifiable. It has been reported that detection of walking speed $<0.8 \mathrm{~m} / \mathrm{s}$ is a single approach to the diagnosis of frailty in the primary care setting [26]. As, in this study, we considered emergency hospital admission and an immobile state as the real-world clinical situation, it is not possible to estimate gait speed in this situation; thus, we assessed RF thickness and activity status of life substitute for gait speed and muscle strength, as indicators of one component of physical frailty. Our study has demonstrated that the frailty scale correlated inversely with RF thickness and activity status of life. Furthermore, before proceeding to 'frail', which was defined as an REFS $\leq 7$, muscle thickness correlated inversely with the frailty scale. RF thickness may be a marker of a high-risk group for whom interventions might be beneficial in preventing further functional decline.

There are several limitations to the current study. Firstly, the subjects were a select population of hospitalized elderly patients with CAP, and sample size is too small to demonstrate that frailty is one of risk factors for CAP. Future investigation of these points will be necessary in a larger size. Secondly, depressive status was not assessed in detail. Depression is the most common cause of unintentional weight loss and undernutrition in the elderly [27]. Thirdly, we measured RF thickness to assess physical functional impairment. In order to assess this, three components, weak muscle strength, slow gait speed, and poor balance [28] should be measured. However, in the situation of the mobility-intact state, the method for detecting physical functional impairment could be limited.

In accelerated aging, it was necessary to estimate the frailty time-window and to prevent disability. Assessment for physical frailty using activity status of life and RF thickness might contribute to predict hospitalized CAP. Future investigation of these factors in the preclinical detection of early manifestations leading to frailty will be useful as predictors of disability, i.e., in elderly CAP using retrospective analysis.

\section{Acknowledgements}

The study was approved by the Yonago Medical Centre Committee on Medical Ethics (25-2). We thank Ms. Asako Fujihara, Ms. Junko Tabuchi, and Ms. Saika Kurumi for their assistance in collecting data as members of the nutrition support team (NST). 


\section{References}

[1] Mandell, L. A., Wunderink, R. G., Anzueto, A., Bartlett, J. G., Campbell, G. D., Dean, N. C., Dowell, S. F., File, Jr. T. M., Musher, D. M., Niederman, A., Torres, N. C., Whitney, C. G.; Infectious Diseases Society of America; American Thoracic Society. Infectious Diseases Society of America/American Thoracic Society Consensus Guidelines on the Management of Community-acquired Pneumonia in Adults. Clin Infect Dis. 2007:44: S27-S72.

[2] Centers for Disease Control and Prevention (CDC). Trends in Aging-United States and World Wide. Morb Mortal Wkly Rep. 2003:52:101-106.

[3] Kaplan, V., Angus D., Griffin, M., Clermont, G., Scott Watson, R., Linde-Zwirble W. Hospitalized Communityacquired Pneumonia in the Elderly: Age- and Sex-related Patterns of Care and Outcome in the United States. Am J Respir Crit Care Med. 2002:165:766-772.

[4] Fried, L. P., Tangen, C. M., Walston, J., Newman, A. B., Hirsch, C., Gottdiener, J., Seeman, T., Tracy, R., Kop, W. J., Burke, G., McBurnie, M. A.; Cardiovascular Health Study Collaborative Research Group. Frailty in Older Adults: Evidence for a Phenotype. J Gerontol A Biol Sci Med Sci. 2006: 56: M146-M156.

[5] Morley, J. E., Haren, M. T., Rolland, Y., Kim, M. J. Frailty. Med Clin North Am. 2006: 90:837-847.

[6] Fried, L. P., Xue, Q. L., Cappola, A. R., Ferrucci, L., Chaves, P., Varadhan, R., Guralnik, J. M., Leng, S. X., Semba, R. D., Walston, J. D., Blaum, C. S., Bandeen-Roche, K. Nonlinear Multisystem Physiological Dysregulation Associated with Frailty in Older Women: Implications for Etiology and Treatment. J Gerontol A Biol Sci Med Sci. 2009: 64: 10491057. doi: $10.1093 /$ gerona/glp076.

[7] Falcone, M., Blasi, F., Menichetti, F., Pea, F., Violi, F. Pneumonia in Frail Older Patients: an Up to Date. Intern Emerg Med. 2012:7:415-424.

[8] Tabue-Teguo, M., Kelaiditi, E., Demougeot, L., Dartigues, J. F., Vellas, B., Cesari, M. Frailty Index and Mortality in Nursing Home Residents in France: Results From the INCUR Study. J Am Med Dir Assoc. 2015: 16:603-606. doi: 10.1016/j.jamda.2015.02.002.

[9] Millett, E. R., De Stavola, B. L., Quint, J. K., Smeeth, L., Thomas, S. L. Risk Factors for Hospital Admission in the 28 days Following a Community-acquired Pneumonia Diagnosis in Older Adults, and their Contribution to Increased Hospitalization Rates Over Time: a Cohort Study. BMJ Open. 2015: 5: e008737. doi: 10.1136/bmjopen-2015-008737.

[10] Sligl, W. I., Majumdar, S. R. How Important is Age in Defining the Prognosis of Patients with Community-acquired Pneumonia? Curr Opin Infect Dis. 2011:24:142-147. doi: 10.1097/QCO.0b013e328343b6f8.

[11] Waters, D., Baumgartner, R. N., Garry, P. J. Sarcopenia: Current Perspectives. J Nutr Health Aging. 2000: 4:133-139.

[12] Chevalier, S., Saoud, F., Gray-Donald, K., Morais, J. A. The Physical Functional Capacity of Frail Elderly Persons Undergoing Ambulatory Rehabilitation is Related to their Nutritional Status. J Nutr Health Aging. 2008:12:721-726.
[13] Studenski, S., Perera, S., Patel, K., Rosano, C., Faulkner, K., Inzitari, M., Brach, J., Chandler, J., Cawthon, P., Connor, E. B., Nevitt, M., Visser, M., Kritchevsky, S., Badinelli, S., Harris, T., Newman, A. B., Cauley, J., Ferrucci, L., Guralnik, J. Gait Speed and Survival in Older Adults. JAMA. 2011:305:50-58. doi: 10.1001/jama.2010.1923.

[14] Podsiadlo, D., Richardson, S. The Timed "Up \& Go": a Test of Basic Functional Mobility for Frail Elderly Persons. $J$ Am Geriatr Soc. 1991:39:142-148.

[15] Beavers, K. M., Beavers, D. P., Houston, D. K., Harris, T. B., Hue, T. F., Koster, A., Newman, A. B., Simonsick, E. M., Studenski, S. A., Nicklas, B. J., Kritchevsky, S. B. Associations between Body Composition and Gait-speed Decline: Results for the Health, Aging, and Body Composition Study. Am J Clin Nutr. 2013: 97:552-560. doi: 10.3945/ajen.112.047860.

[16] Takai, Y., Ohta, M., Akagi, R., Kato, E., Wakahara, T., Kawakami, Y., Fukunaga, T., Kanehisa, H. Validity of Ultrasound Muscle Thickness Measurements for Predicting Leg Skeletal Muscle Mass in Healthy Japanese Middle-aged and Older Individuals. J Physiol Anthropol. 2013:32:12.

[17] Fried, L. P., Ferrucci, L., Darer, J., Williamson, J. D., Anderson, G. Untangling the Concepts of Disability, Frailty, and Comorbidity: Implications for Improved Targeting and Care. J Gerontol A Biol Sci Med Sci. 2004: 59:255-263.

[18] Roy, C. N. Anemia in Frailty. Clin Geriatr Med. 2011: 27:67-78.

[19] Lim, W. S., Baudouin, S. V., George, R. C., Hill, A. T., Jamieson, C., Le Jeune, I., Macfarlane, J. T., Read, R. C., Roberts, H. J., Levy, M. L., Wani, M., Woodhead, M. A.; Pneumonia Guidelines Committee of the BTS Standards of Care Committee. BTS Guidelines for the Management of Community Acquired Pneumonia in Adults: Update 2009. Thorax. 2009: 64: iii1-iii55. doi: 10.1136/thx.2009.121434.

[20] Hilmer, S. N., Perera, V., Mitchell, S., Murnion, B. P., Dent, J., Bajorek, B., Matthews, S., Rolfson, D. B. The Assessment of Frailty in Older People in Acute Care. Australas J Ageing. 2009:28:182-188. doi: 10.1111/j.1741-6612.2009.00367.x.

[21] De Bruin, P. F., Ueki, J., Watson, A., Pride, N. B. Size and Strength of the Respiratory and Quadriceps Muscles in Patients with Chronic Asthma. Eur Respir J. 1997:10:59-64.

[22] Obuchi, S., Aria, T., Kojima, M., Kawai, H., Kojima, N. Relationship between Knee Extension Strength and the Quadriceps Femoris Muscle Thickness Measured by Ultrasound. Rigakuryoho Kagaku. 2009:24:185-190 [Japanese].

[23] Oken, M. M., Creech, R. H., Tromey, D. C., Horton, J., Davis, T. E., McFadden, E. T., Carbone, P. P. Toxicity and Response Criteria of the Eastern Cooperative Oncology Group. Am J Chin Oncol 1982: 5:649-655.

[24] Guigoz, Y., Vellas, B., Garry, P. J. Assessing the Nutritional Status of the Elderly: the Mini Nutritional Assessment as Part of the Geriatric Evaluation. Nutr Rev. 1996: 54: S59-65.

[25] Draper, N., Smith, H. (1981) Applied Regression Analysis. 2nd ed. John Wiley \& Sons Inc, New York.

[26] Castell, M. V., Sánchez, M., Julián, R., Queipo, R., Martín, S., Otero, Á. Frailty Prevalence and Slow Walking Speed in Persons Age 65 and Older: Implications for Primary Care. BMC Fam Pract. 2013:14:86. 
[27] Evers, M. M., Marin, D. B. Mood Disorders. Effective Management of Major Depressive Disorder in the Geriatric Patient. Geriatrics. 2002:57:36-40.
[28] Cesari, M., Landi, F., Vellas, B., Bernabei, R., Marzetti, E. Sarcopenia and Physical Frailty: Two Sides of the Same Coin. Front Aging Neurosci. 2014:6:192. 